\title{
PERFILES DE DIFÍCIL CONTRATACIÓN Y NECESIDADES DE CAPACITACIÓN: UNA VISIÓN DE LAS EMPRESAS DE RECLUTAMIENTO
}

\author{
HIRING DIFFICULT PROFILES AND TRAINING NEEDS: \\ A VISION OF PERSONNEL RECRUITMENT FIRMS
}

Byron Acosta Doctor en Administración (Universidade Federal do Rio Grande do Sul/Brasil). Profesor de la Universidad Técnica del Norte - UTN (Ibarra/Ecuador). E-mail:byron@hotmail.com.br.

Verónica García Maestra en Psicología (Pontificia Universidad Católica del Ecuador/Ecuador). Profesora en la Pontificia Universidad Católica del Ecuador (Quito/Ecuador). E-mail.vgarciao@puce.edu.ec.

\section{Miguel Acosta}

Maestro en Economía (Universidad Autónoma de México/México).

Profesor en la Pontificia Universidad Católica del Ecuador (Quito/Ecuador) E-mail.vgarciao@puce.edu.ec. 


\section{RESUMEN}

El objetivo de esta investigación es identificar los perfiles de difícil contratación y las necesidades de capacitación profesional sobre la perspectiva de organizaciones de reclutamiento de personal. Fue aplicada una encuesta a 12 empresas de selección y reclutamiento de personal. Los resultados fueron que, para las empresas especializadas en selección de personal, las organizaciones que más demandan sus servicios se agrupan en los sectores de Alimentos frescos y procesados; Construcción; Tecnología en hardware y software; y Productos farmacéuticos y químicos. En segundo lugar, los sectores productivos con alta perspectiva de empleabilidad son los siguientes: Tecnología en hardware y software; Vehículos, automotores, carrocerías y partes; Turismo; Construcción; y Energías renovables. Igualmente, las nuevas ocupaciones y las que tienen dificultades deencontrartrabajadores competentes son: Profesionalescientíficos eintelectuales; Trabajadores de los servicios y vendedores de comercios y mercados; y profesionales de nivel medio. Finalmente, las áreas académicas de mayor demanda o que se requiere de personal fueron identificadas como: Administración y legislación; Educación y Capacitación y Tecnologías de la información y comunicación.

Palabras-clave: Necesidades de capacitación. Perfil. Difícil contratación.

\section{ABSTRACT}

The objective of this research was to identify training areas prioritized by the productive sectors in Ecuador. It was developed a methodology that was used to implement a continuous process of identifying training needs of productive sectors at zonal and national levels. Companies specializing in recruitment human resources indicate that most organizations claim their services are grouped into sectors of Food, Construction, Technology, and Pharmaceuticals. Second, the productive sectors with high employability perspective are: Technology, vehicles and parts, Tourism, Construction, and Energy. Similarly, occupations who are struggling to find skilled workers are scientific professionals, service workers and salesmen and professional level. Finally, the academic areas of greatest demand or personnel required were identified as: Administration and legislation, Training and Information Technology and Communication.

Keywords: Professional development needs. Profile. Difficult occupation. 


\section{INTRODUCCIÓN}

Por un lado, el desarrollo y la introducción de nuevos productos al mercado, obliga a las empresas a crear nuevas ocupaciones que fusionan áreas del conocimiento diferentes. Por ejemplo, la ingeniería mecánica se une con la eléctrica para crear la meca-trónica, o la aplicación de principios y técnicas de la ingeniería en la medicina se consolida como la ingeniería biomédica. Es decir, la solución a problemas o la satisfacción de las necesidades de las personas demanda la creación de nuevos puestos de trabajo que antes no existían o que las empresas no preveían su adopción.

Por otro lado, la falta de capacitación en las empresas ocasiona problemas de productividad, baja calidad en los productos, uso deficiente de la maquinaria, desperdicio de materiales y accidentes laborales, entre otros. Estos problemas se potencializan cuando las organizaciones desarrollan procesos de innovación sin realizar paralelamente acciones de formación profesional. Precisamente, debido a la acelerada absorción de conocimientos requerida y a la falta de entrenamiento; el recurso humano de las organizaciones necesita adaptarse inmediatamente al uso de herramientas, equipos, y mejores prácticas; surgiendo necesidades de capacitación a ser satisfechas.

La modernización de la gestión empresarial y la capacitación del personal son dos elementos que deben realizarse de forma simultánea. La innovación tecnológica por medio de la adquisición de nuevos equipos debe realizarse en forma paralela con la capacitación del personal de la organización (INA, 2007). En este sentido, la adquisición de equipos de alta tecnología no garantiza a la empresa resultados positivos, si el talento humano no cuenta con los conocimientos y habilidades necesarios para operar dichos equipos o aplicar nuevos conocimientos. Según Ortega (2006, p. 1) "Hoy se considera que cada ocho meses los individuos tienen que estar en recapacitación para estar al día en las exigencias del mercado, de las empresas".

Para identificar las necesidades de capacitación del recurso humano, se debe conocer en qué medida la persona está consciente de sus necesidades objetivas, y relacionarlas con sus intereses en formación y entrenamiento. Además, se requiere identificar exactamente el perfil de los beneficiarios a los cuales estarán dirigidos los procesos formativos (ADRIÁN; PAULA, 1992). Según Mendoza (1996), las metodologías para determinar las necesidades de capacitación y formación deben integrar tres componentes básicos: las políticas y lineamientos institucionales, la situación actual de recurso humano, y la planificación del talento humano a nivel local, regional y por sectores de la economía.

Con base en esa discusión surge la siguiente pregunta: ¿Cuáles son las nuevas ocupaciones y las necesidades de capacitación y formación profesional que las empresas necesitan? Es así que el objetivo de esta investigación es identificar las nuevas ocupaciones y las necesidades de capacitación y formación profesional en las empresas. 
El artículo está estructurado de la siguiente forma: Primero, el marco teórico presentando una discusión sobre levantamiento de necesidades de capacitación y formación profesional. Segundo, los procedimientos metodológicos aplicados en cuatro empresas de reclutamiento y selección de personal. Tercero, el análisis de resultados y discusión de los datos. Finalmente las conclusiones, limitaciones y propuestas para futuras investigaciones.

\section{2 rEVISIÓN DE LA LITERATURA}

\subsection{LA CAPACITACIÓN Y EL LEVANTAMIENTO DE NECESIDADES DE CAPACITACIÓN EN LAS ORGANIZACIONES}

Desde la década de los 70, el recurso humano enfrenta exigencias debido a la competitividad laboral y a la rápida evolución tecnológica, lo que obligó a las empresas a acelerar los procesos de aprendizaje (DA SILVA; DA CONCEIÇÃO; LEITE, 2008). Incluso en esa década se menciona la pertinencia del convenio 42 en la conferencia general de la Organización Internacional del Trabajo (OIT) donde se señala que los países miembros deben mantener sistemas flexibles para la formación técnica y profesional tanto dentro del sistema educativo como fuera de éste (LACAVEX, 2013). Lo que generó que exista la necesidad de incorporar mayor información sobre el empleo en el sector educativo, y esto a su vez se traduce en que las organizaciones se agilicen e incorporen entrenamiento al interior de la organización, en el puesto, de tal manera que los países inicien con normativas hacia la obligación por parte de los empleadores para proveer de adiestramiento a sus trabajadores (LACAVEX, 2013).

Según Bomfin (2004), existen dos visiones sobre las cuales puede ser observada la capacitación; en la visión tradicional, la capacitación no considera las habilidades intelectuales ni la condición humana de los empleados, lo que se asemeja al adiestramiento anteriormente mencionado; ya en la visión moderna, la capacitación busca el desarrollo de competencias del ser humano.

Para Cruz (1997), las empresas consideran que los procesos de entrenamiento deben ser alineados al cumplimiento de los objetivos de la organización. Boog (1994), destaca que el entrenamiento y la capacitación empresarial se originan del deseo de reducir los riesgos de accidentes de trabajo, bajar los daños en los equipos, disminuir los desperdicios, y aumentar la producción y la calidad de productos. Sobre este tema existen sendos debates para ubicar a la formación profesional y la capacitación en el campo educativo o productivo (VERDIER, 2008). Si se la ubica en este último se entiende que debe existir un diálogo sobre el enfoque de los programas de formación, entre el Estado, la organización, y el trabajador, y en algunos casos incluso el sindicato (JACINTO, 2015). 
Desde este enfoque productivo, uno de los factores a ser tomados al escoger programas de capacitación es el giro de negocio de la empresa. Según Lefcovich (2009), la capacitación puede ser desarrollada a través de programas de formación profesional, perfeccionamiento y/o capacitación. Así, los niveles de preparación que las empresas demandan deben utilizar métodos de enseñanza que deben ser considerados al momento de elegir un programa de capacitación. Dalmau, Valente, Lobo, Barcia y Nascimento (2001), señalan que el entrenamiento puede ser presencial, semi-presencial o a distancia. Siendo el primero el más popular y el tercero el de mayor crecimiento en demanda (DALMAU et al., 2001).

Según Dalmau et al. (2001), los factores que determinan la decisión del tipo de capacitación son el tiempo que dure la capacitación, el costo, el tamaño de la demanda del curso, la metodología y el elemento humano participante. Para Harris (2006), los criterios que la empresa prioriza para capacitarse son: si el tema es de su interés, cuando y donde será impartido, y si lo costo será o no pagados por el empleador. Se observa que el costo es el factor común para los dos autores en el momento de decidir el tipo de capacitación en que optaran las empresas.

Identificados los factores que determinan el tipo de capacitación, la segunda etapa es determinar los temas en que deben ser capacitados los trabajadores; es decir, determinar sus necesidades de capacitación. Las necesidades de capacitación son las carencias profesionales de información, conocimientos, habilidades y actitudes detectadas en las personas cuando no alcanzan los niveles de competencias mínimos exigidos para el desempeño eficiente en una ocupación o puesto de trabajo (SERPE, 2006).

Las necesidades de capacitación llevan en consideración a los equipos y maquinaria disponibles, al nivel de tecnología empleada, al tipo de organización, y la actividad que desempeña el recurso humano dentro de la empresa (BARROS, 2011). De esta forma las necesidades de capacitación son causa, cuando el conocimiento se encuentra incorporado en las personas y son consecuencia, cuando se demanda recursos humanos calificados debido a la mayor complejidad tecnológica (MEJÍA; MIRANDA; QUIÑONES, 2004).

\subsection{EL LEVANTAMIENTO DE NECESIDADES DE CAPACITACIÓN EN LOS PROGRAMAS DE CAPACITACIÓN}

Reconocido el hecho de que la estrategia empresarial exige el planteamiento de un programa de capacitación, el primer paso a seguir es identificar las necesidades de capacitación para elaborar programas de entrenamiento y formación profesional; es decir, identificar las discrepancias entre lo que es y lo que debería ser (POR; AGUILAR, 2010). El levantamiento de necesidades constituye la primera fase del ciclo de formación, y determina todas las fases subsecuentes del proceso de capacitación dentro de la organización (POR; AGUILAR, 2010). 
El levantamiento de necesidades tiene como fin, identificar las brechas de entre las competencias presentadas por el trabajador y las definidas en el perfil duro que son indispensables para asumir el cargo, y que impactará en la consecución de sus objetivos organizacionales. Según Moraes (2002) en esta etapa se identifica quien deberá ser capacitado y en qué contenidos hacerlo, para cubrir la diferencia entre lo que existe en términos de conocimiento dentro de la organización y el óptimo deseado para el futuro. Como McGehee y Thayer (1961) explican, una organización debe realizar el levantamiento de necesidades de capacitación en tres niveles: análisis organizacional, análisis de las tareas y análisis del individuo.

Para Cruz (1997), el análisis organizacional identifica los vacíos de formación profesional que afectan a la consecución de los objetivos estratégicos de la empresa. Este análisis está atado directamente a los planteamientos estratégicos de la organización. En la misma línea, Cruz (1997) define al análisis de tareas como la manera de identificar la naturaleza de las diferentes tareas para el mejor desempeño de puesto. De acuerdo con Gil (2001), este análisis requiere la obtención de los siguientes datos: a) identificación de las actividades que componen la tarea, b) identificación de las responsabilidades de los ejecutores de la tarea, c) identificación de las condiciones del trabajo y riesgos y d) conocimientos, habilidades y actitudes requeridas. Por último, El mismo autor indica que a través del análisis del individuo, se identifican a los empleados que tienen la motivación, el interés, las actitudes y los conocimientos que puedan maximizar los beneficios de la capacitación.

\subsection{TÉCNICAS DE LEVANTAMIENTO DE NECESIDADES DE CAPACITACIÓN}

Dentro de las técnicas para realizar el levantamiento de necesidades de capacitación se encuentran: la revisión de los análisis de puestos, el análisis de inventario de habilidades de la empresa, el análisis del desempeño de los trabajadores, quejas de los clientes, el análisis de problemas de la empresa, exámenes de conocimientos, entrevistas estructuradas, encuestas, observación directa en el puesto, análisis de tareas, entre otras (POR; AGUILAR, 2010).

Moraes (2002) indica que el análisis de documentos, la realización de entrevistas y encuestas, las entrevistas por salida de personal, y reuniones con la alta gerencia son los instrumentos más comunes para levantar la información en primera instancia. Sobre la base de estos instrumentos y previo al análisis de la información levantada se puede determinar las necesidades de capacitación en cana nivel jerárquico.

Según Meneses y Zerbini (2010), el éxito de todo programa de capacitación depende de la realización de un levantamiento sistemático de las necesidades de capacitación, a través de los tres análisis básicos organizacional, tareas e individuo-. El levantamiento de necesidades conforma las directrices para el proceso de evaluación de la capacitación y evaluación del desempeño del empleado; con el fin de determinar los 
beneficios para los trabajadores en la organización, el aumento en la remuneración, mayor responsabilidad en el cargo, promociones, nuevas oportunidades de trabajo, entre otras (HARRIS, 2000).

\section{METOdOLOGÍA}

El objetivo de esta investigación fue identificar los perfiles de difícil contratación y las necesidades de capacitación profesional sobre la perspectiva de organizaciones de reclutamiento de personal. Así, en cuanto a su alcance, este estudio es de carácter exploratorio y de enfoque cuantitativo. Una encuesta fue aplicada a una muestra de 40 empresas de reclutamiento y selección de personal en el Ecuador, teniendo como universo 117 empresas de este tipo. De la muestra de empresas, 15 respondieron el cuestionario, lo que significa una tasa de respuesta del $38 \%$. Posteriormente se realizó la depuración de las encuestas, de las cuales tres fueron descartadas por tener la información incompleta. Así, el análisis se realizó con datos de 12 empresas que corresponden al 30\% de la muestra seleccionada.

El instrumento utilizado para el levantamiento de la información fue un cuestionario on-line. Las preguntas que integran el instrumento se presentan en el Cuadro 1. Antes de aplicar la encuesta, la misma fue examinada por expertos en gestión de recursos humanos, con el objetivo de verificar la validez del instrumento y la confiabilidad de los datos a ser recolectados. Finalmente, los datos fueron analizados por medio de estadística descriptiva.

\section{Cuadro 1 - Preguntas instrumento de recolección de datos}

1. ¿En qué sectores económicos actúan sus clientes más recurrentes?

2. ¿Cuál es el nivel mínimo de formación que solicitan sus clientes (empresas) para puestos técnicos?

3. ¿Cuál es el nivel mínimo de formación que solicitan sus clientes (empresas) para puestos administrativos?

4. ¿Para sus clientes (empresas), cuál es la perspectiva de generación de empleo para el año en curso?

5. ¿En qué sectores considera Usted que existirá un crecimiento significativo en la generación de empleo para el año en curso?

6. Para sus clientes (empresas), cuáles son los meses de mayor contratación de personal?

7. ¿Para sus clientes (empresas), cuáles son las ocupaciones en que existen previsiones de contratación?

8. ¿En qué ocupaciones existen dificultades de encontrar trabajadores calificados?

9. ¿Cuál es la causa que más dificulta la contratación de trabajadores?

10. ¿En base a los requerimientos de sus clientes, cuáles son las nuevas ocupaciones que están siendo solicitadas?

11. ¿En qué temas específicos deberían estar capacitados los candidatos para que puedan ser contratados?

\section{Fuente: elaborado por los autores}




\section{RESULTADOS Y ANÁLISIS}

\subsection{DATOS TÉCNICOS DE LAS ORGANIZACIONES}

Las reclutadoras de personal tienen como clientes más recurrentes las empresas de Alimentos frescos y procesados; Construcción; Tecnología en hardware y software, y Productos farmacéuticos (Gráfico 1). Con excepción del sector de Hardware y Software, los resultados muestran que las empresas del sector de transformación y producción de bienes demandan más recurso humano, en relación a las de servicio y comercialización.

Gráfico 1 - Clientes por sector económico

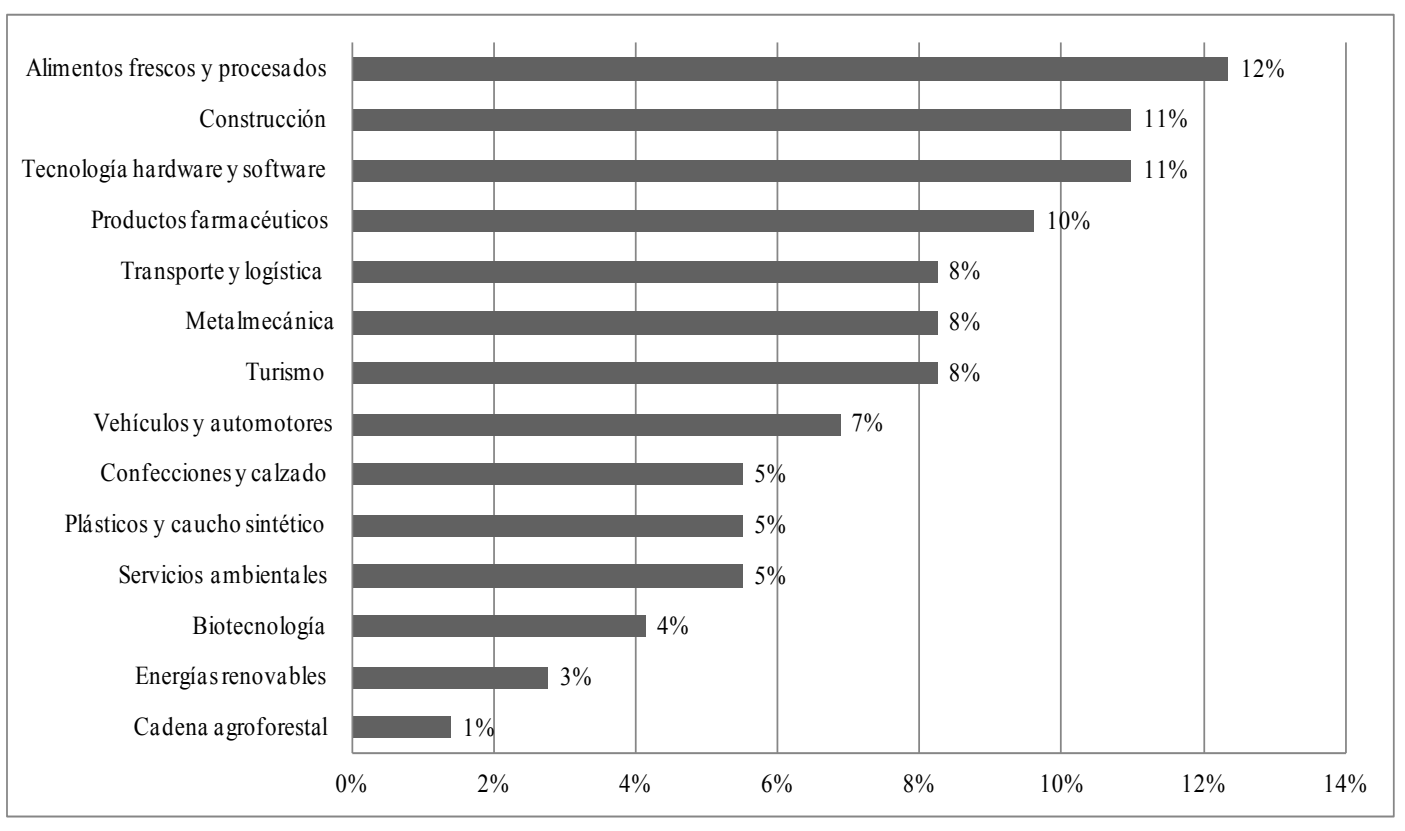

Fuente: elaborado por los autores

Referente al nivel mínimo de escolaridad que las empresas necesitan para cubrir sus puestos de trabajo. Según las reclutadoras de personal (Gráfico 2), para puestos técnicos, el mínimo requerido corresponde a un nivel técnico (29\%) y formación universitaria (tercer nivel) (22\%); ya para cargos administrativos, el nivel mínimo requerido es la formación universitaria (57\%). Es fundamental que la formación educativa-profesional y el perfil del cargo sean equivalentes los puestos técnicos al ser orientados a un contexto industrializado donde priman las actividades rutinarias, en trabajos de difícil movilidad y más precariedad, con sueldos inferiores a los que se estiman en los cargos administrativos (PIECK GOCHICOA, 2011). Además, se requiere de 
una formación de corta duración, masiva y que le permita al trabajador un ingreso rápido al mercado laboral, esta educación técnica puede darse a través de institutos de formación técnica o capacitaciones con cursos cortos que preparan para oficios concretos (PIECK GOCHICOA, 2011).

\section{Gráfico 2 - Mínimo de escolaridad para vacantes}

Puestos técnicos

\section{Fuente: elaborado por los autores}

En el caso de cargos administrativos prima la formación universitaria donde los trabajadores deben presentar competencias blandas en mayor nivel de desarrollo y la educación universitaria procura la adquisición de las mismas aunque sea en un grado inicial dentro de su educación integral, pues "la Universidad es la cantera de donde saldrán los líderes sociales, económicos y políticos que gobernarán el país" (RAMOS, 1991, p. 323).

\subsection{ESTIMACIÓN DEL SISTEMA LABORAL}

Respecto a la perspectiva de generación de empleo, el 50\% de las reclutadoras estiman un crecimiento estable y 22\% esperan un crecimiento moderado, para el segundo semestre del 2012 (Gráfico 3). Esos resultados muestran una oportunidad para emprender acciones de capacitación y formación profesional orientadas a satisfacer la demanda de talento humano en las áreas priorizadas. De hecho, para Rodríguez (2003, p. 278) “la capacitación incrementa de 20 a 60 \% la probabilidad de encontrar un empleo"; y en función de la modalidad de capacitación incluso se ha visto que incide en la posibilidad de salir del desempleo, de tal forma que la capacitación con modalidad escolarizada (cuando las capacitaciones son totalmente gestionadas por el centro de formación) permite que existe un 20 \% de probabilidad de salir del desempleo; mientras que la modalidad mixta (cuando en las capacitaciones participan las empresas a través de aportes de materiales y del compromiso de seleccionar a un número de personas participantes del programa de formación) es del 60 \% (RODRÍGUEZ, 2003). 


\section{Gestãoe \\ Desenvolvimento}

Gráfico 3 - Grado de generación de empleo

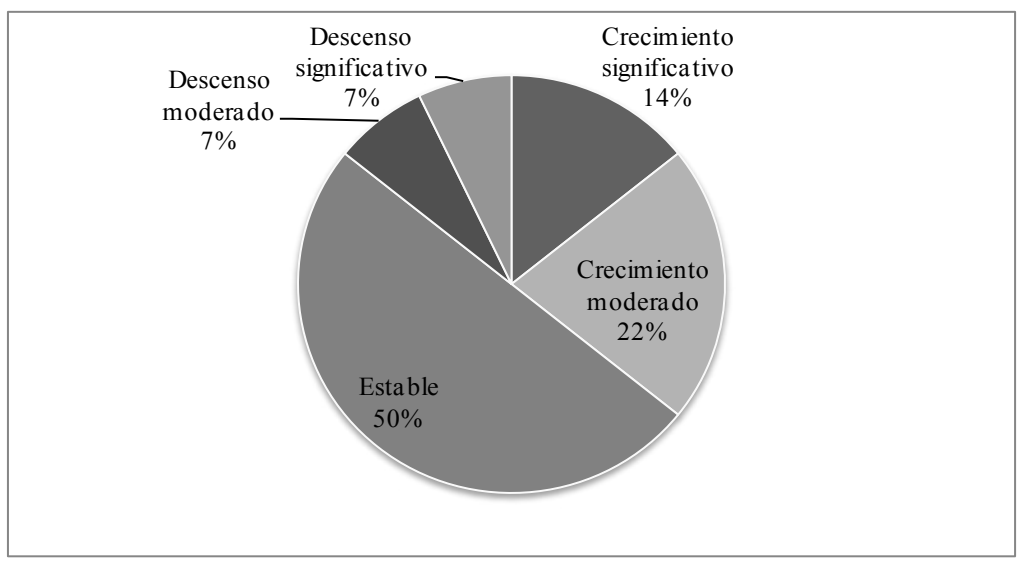

Fuente: elaborado por los autores

Para las empresas de selección de personal, los sectores productivos que tendrán un crecimiento significativo en la generación de empleo son (Gráfico 4): Tecnología en hardware y software; Vehículos, automotores, carrocerías y partes; Turismo; Construcción; y Energías renovables.

\section{Gráfico 4 - Demanda de RRHH por sector}

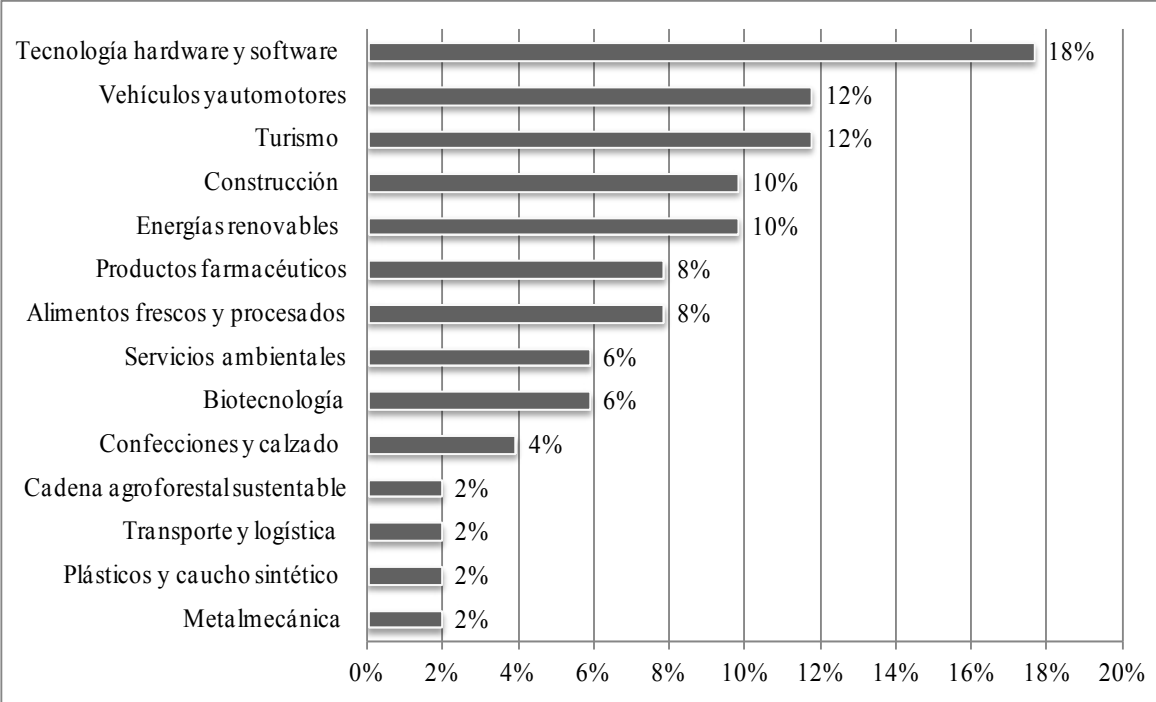

Fuente: elaborado por los autores 


\section{Gestãoe \\ Desenvolvimento}

\subsection{USO Y PERFIL DEL SERVICIO}

Una vez identificados los sectores productivos en que se deberán priorizar los procesos formativos, la siguiente etapa es direccionar la capacitación a las ocupaciones que tienen mayor previsión de contratación. Para las empresas de selección de personal, las ocupaciones que tienen alta previsión de contratación son: Trabajadores de los servicios y vendedores de comercios y mercados; Personal de apoyo administrativo; y Técnicos y profesionales de nivel medio (Gráfico 5). Sin embargo, a nivel de Directores y gerentes, y Ocupaciones elementales se debe también considerar como una segunda prioridad para direccionar la oferta formativa de instituciones públicas o privadas. Además, los meses de año más adecuados para organizar procesos de formación profesional son enero, abril y agosto. El Ecuador mantiene estrategias para el cambio de la matriz productiva, como se evidencia desde hace décadas, los talentos altamente calificados, a nivel científico e intelectual, se encuentra en la academia y no en el sector productivo "profesionales de nivel medio se concentran en su mayoría en el sector de educación y administración pública, mientras que las industrias manufactureras agrupan menos del 6\% de estos profesionales" (VICEPRESIDENCIA DE LA REPÚBLICA DEL ECUADOR, 2015, p. 31), pero actualmente se desea transformar esa condición laboral para que se contemple la conjunción entre la parte educativa y la formación profesional con el mercado laboral.

Gráfico 5 - Ocupaciones que demandan capacitación

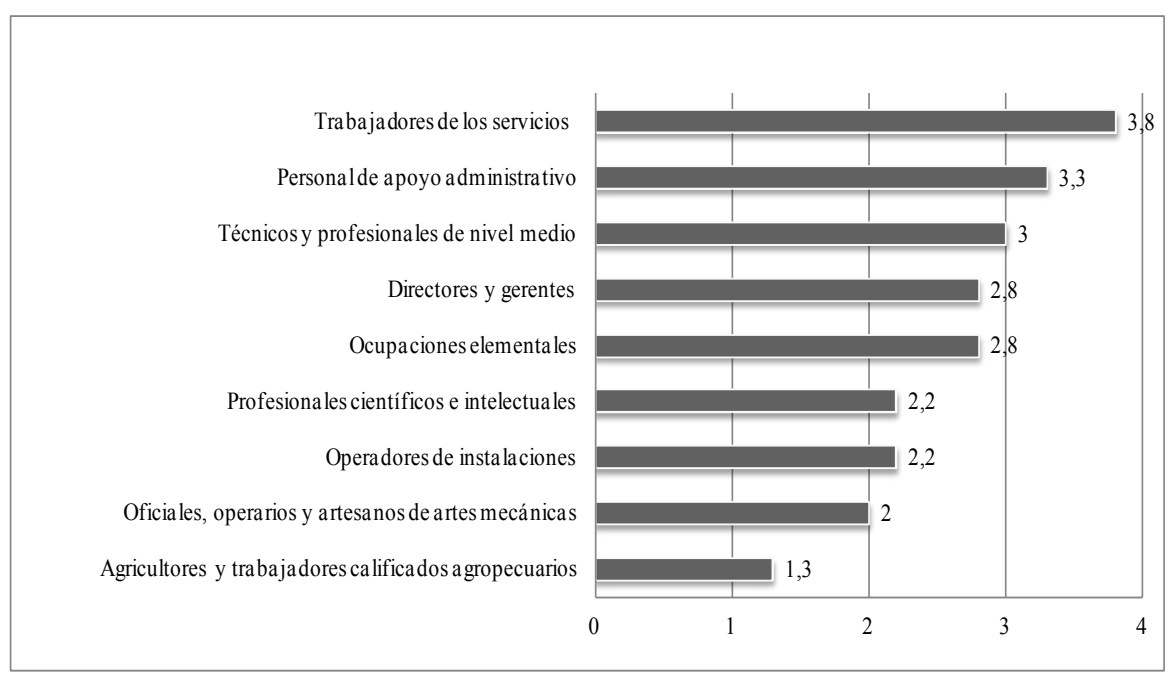

Fuente: elaborado por los autores 


\subsection{NECESIDADES DE CAPACITACIÓN}

Para determinar las necesidades reales de capacitación de los representantes de dicho sector, se identificaron los principales factores que dificultan la inserción de los individuos en el sistema laboral. Los encuestados señalan, en primer lugar, el bajo nivel académico, la falta de personal calificado y la falta de formación profesional, como los motivos que más dificultan su acceso a fuentes estables de trabajo. En segundo lugar, la falta de capacitación específica; los horarios de trabajo y la falta de experiencia práctica en el trabajo, se posicionan igualmente como factores que obstaculizan el acceso a fuentes de trabajo.

Las empresas de selección de personal encuestadas tienen dificultades de encontrar trabajadores competentes principalmente en las ocupaciones relacionadas con: Profesionales científicos e intelectuales, Trabajadores de los servicios y vendedores de comercios; Técnicos y profesionales de nivel medio. Además, los resultados muestran que hay ocupaciones que pueden desempeñar personas con discapacidad, donde se refleja escasez de talento humano. El Cuadro 2 presenta una síntesis de las ocupaciones con dificultades de contratación, agrupada de acuerdo a la Clasificación Internacional Uniforme de Ocupaciones (CIUO-88) de grandes grupos y grupos primarios.

Cuadro 2 - Ocupaciones de difícil contratación

(continua)

\begin{tabular}{|c|c|}
\hline Grandes grupos & Grupos primarios \\
\hline Directores y gerentes & $\begin{array}{l}\text { Directores generales y gerentes generales } \\
\text { Directores financieros }\end{array}$ \\
\hline Profesionales científicos e intelectuales & $\begin{array}{l}\text { Profesionales de la salud y la higiene laboral y ambiental } \\
\text { Contables } \\
\text { Profesionales de ventas de tecnología de la información y las comu- } \\
\text { nicaciones } \\
\text { Analistas de sistemas } \\
\text { Programadores de aplicaciones } \\
\text { Profesionales del trabajo social } \\
\text { Traductores, intérpretes y lingüistas }\end{array}$ \\
\hline Técnicos y profesionales de nivel medio & $\begin{array}{l}\text { Técnicos en ciencias físicas y químicas } \\
\text { Técnicos en electrónica } \\
\text { Técnicos en ingeniería mecánica } \\
\text { Profesionales de nivel medio de enfermería } \\
\text { Representantes comerciales } \\
\text { Técnicos en operaciones de tecnología de la información y las comu- } \\
\text { nicaciones } \\
\text { Técnicos en asistencia al usuario de tecnología de la información y } \\
\text { las comunicaciones } \\
\text { Técnicos en redes y sistemas de computadores }\end{array}$ \\
\hline
\end{tabular}


(continuação)

\begin{tabular}{|l|l|}
\hline Personal de apoyo administrativo & $\begin{array}{l}\text { Empleados de contabilidad y cálculo de costos } \\
\text { Empleados de servicios de transporte } \\
\text { Empleados del servicio de personal }\end{array}$ \\
\hline $\begin{array}{l}\text { Trabajadores de los servicios y vende- } \\
\text { dores de comercios y mercados }\end{array}$ & $\begin{array}{l}\text { Vendedores } \\
\text { Comerciantes de tiendas } \\
\text { Asistentes de venta de tiendas y almacenes }\end{array}$ \\
\hline $\begin{array}{l}\text { Oficiales, operarios y artesanos de artes } \\
\text { mecánicas y de otros oficios }\end{array}$ & $\begin{array}{l}\text { Electricistas de obras y afines } \\
\text { Mecánicos y reparadores en electrónica } \\
\text { Costureros, bordadores y afines }\end{array}$ \\
\hline $\begin{array}{l}\text { Operadores de instalaciones y máquinas } \\
\text { y ensambladores }\end{array}$ & \begin{tabular}{l} 
Operadores de plantas y máquinas de productos químicos \\
\hline Ocupaciones elementales
\end{tabular} \\
\hline Otros no clasificados & Ocupaciones varias para personal con discapacidad \\
\hline
\end{tabular}

\section{Fuente: elaborado por los autores}

De acuerdo a las empresas de selección de personal, las nuevas ocupaciones que demandan las empresas son: Profesionales científicos e intelectuales, relacionadas con ingenierías, contabilidad y tecnologías de la información, entre otras. La segunda se relaciona con Técnicos y profesionales de nivel medio de las áreas de física, química, electrónica y derecho principalmente. Adicionalmente buscan especialistas en Business Intelligence, expertos en Business Process Management (BPM), ocupaciones para discapacitados y perfiles con conocimientos bilingües. El Cuadro 3 una síntesis de las nuevas ocupaciones que demanda las empresas, agrupadas por la clasificación CIUO-88 de grandes grupos y grupos primarios.

Cuadro 3 - Nuevas ocupaciones demandadas

(continua)

\begin{tabular}{|l|l|}
\hline Grandes grupos & Grupos primarios \\
\hline \multirow{3}{*}{ Directores y gerentes } & Directores generales y gerentes generales \\
& Directores financieros \\
& Directores de industrias manufactureras \\
\hline \multirow{5}{*}{ Profesionales científicos e intelectuales } & Ingenieros medioambientales \\
& Ingenieros químicos \\
& Ingenieros electrónicos \\
& Profesionales de enfermería \\
& Contables \\
& Analistas financieros \\
& Administradores de sistemas \\
\hline
\end{tabular}


(continuação)

\begin{tabular}{|l|l|}
\hline \multirow{2}{*}{ Técnicos y profesionales de nivel medio } & $\begin{array}{l}\text { Técnicos en ciencias físicas y químicas } \\
\text { Técnicos en electrónica } \\
\text { Técnicos en química industrial } \\
\text { Representantes comerciales } \\
\text { Profesionales de nivel medio del derecho y servicios legales y afines }\end{array}$ \\
\hline \multirow{2}{*}{$\begin{array}{l}\text { Personal de apoyo administrativo } \\
\text { vendedores de comercios y mercados }\end{array}$} & $\begin{array}{l}\text { Empleados de contabilidad y cálculo de costos } \\
\text { Empleados de servicios de transporte } \\
\text { Empleados del servicio de personal }\end{array}$ \\
\hline $\begin{array}{l}\text { Oficiales, operarios y artesanos de artes } \\
\text { mecánicas y de otros oficios }\end{array}$ & \begin{tabular}{l} 
Mecánicos y reparadores de vehículos de motor \\
\hline Ocupaciones elementales
\end{tabular} \\
\hline \multirow{2}{*}{\begin{tabular}{l} 
Limpiadores y asistentes domésticos \\
\hline
\end{tabular}} & $\begin{array}{l}\text { Botones Bilingües } \\
\text { Especialistas en Business Intelligence } \\
\text { Expertos en Business Process Management (BPM) } \\
\text { Ocupaciones varias para personal con discapacidad } \\
\text { Secretarias bilingües }\end{array}$ \\
\hline
\end{tabular}

\section{Fuente: elaborado por los autores}

Finalmente, el recurso humano de difícil contratación y los candidatos de nuevas ocupaciones deberán estar capacitados en las siguientes áreas académicas: Administración y legislación; Tecnologías de la información y comunicación; Finanzas, comercio y ventas; Educación y capacitación. Existen ocupaciones de temas relacionados con Biología, Business intelligence, Cultura general y Planificación estratégica, que deben ser considerados para procesos de entrenamiento o capacitación específica. Sin embargo, el área de mayor requerimiento es Administración del talento humano. El Cuadro 4 presenta una síntesis de las especialidades de capacitación y formación profesional agrupadas por áreas académicas. 
Cuadro 4 - Temas de capacitación y formación profesional

\begin{tabular}{|c|c|}
\hline Áreas académicas & Especialidades \\
\hline Administración y legislación & $\begin{array}{l}\text { Administración general (pública, empresas, microempresas, cooperativas, } \\
\text { aduanera, agrícola, agropecuaria, agroindustrial, bancaria, financiera, forestal, } \\
\text { hospitalaria, hotelera, inmobiliaria, pesquera, minera, etc.). } \\
\text { Administración del talento humano (manejo de personal, desempeño, } \\
\text { motivación, liderazgo, coaching, trabajo en equipo) } \\
\text { Administración contable y de costos (matemáticas, estadística, tributaria, } \\
\text { normas de contabilidad, auditorías financieras, contables, de costos y } \\
\text { relacionadas). } \\
\text { Atención y servicios de oficina: secretariado (operación de máquinas de oficina, } \\
\text { taquigrafía, lectura rápida, oratoria, redacción y ortografía), recepción, servicio } \\
\text { al cliente, archivo, conserjería, guardianía, limpieza. } \\
\text { Administración de la calidad (normas, auditorías de sistemas de calidad y } \\
\text { mejoramiento continuo). }\end{array}$ \\
\hline $\begin{array}{l}\text { Tecnologías de la información } \\
\text { y comunicación }\end{array}$ & $\begin{array}{l}\text { Telecomunicaciones (comunicación telefónica, telegráfica, satelital). } \\
\text { Hardware y equipos (arquitectura de pc, mantenimiento, configuración). } \\
\text { Programas de escritorio (office, hojas electrónicas, procesadores de texto, } \\
\text { power point). } \\
\text { Lenguaje de programación (pascal, basic, cobol, visual basic, c+++, power } \\
\text { builder, clipper, java, php, puntonet). }\end{array}$ \\
\hline Finanzas, comercio y ventas & $\begin{array}{l}\text { Marketing y ventas (negociación, comercialización, marketing y ventas de } \\
\text { productos y servicios) }\end{array}$ \\
\hline Educación y capacitación & $\begin{array}{l}\text { Capacitación (identificación de necesidades, procesos de capacitación continua } \\
\text { y por competencias laborales, evaluación y seguimiento). } \\
\text { Formación de instructores, facilitadores, monitores, maestros, guías, } \\
\text { formadores. } \\
\text { Medios y materiales didácticos (diseño, elaboración). }\end{array}$ \\
\hline Electricidad y electrónica & $\begin{array}{l}\text { Electricidad domiciliaria e industrial (reparación, manejo y mantenimiento). } \\
\text { Electromecánica (instalación y mantenimiento de motores eléctricos). }\end{array}$ \\
\hline Comunicación y artes gráficas & Idiomas \\
\hline Otros no clasificados & $\begin{array}{l}\text { Biología } \\
\text { Especialistas Business intelligence } \\
\text { Cultura general } \\
\text { Planificación estratégica }\end{array}$ \\
\hline
\end{tabular}

Fuente: elaborado por los autores 


\section{CONSIDERACIONES FINALES}

El objetivo de esta investigación fue identificar los perfiles de difícil contratación y las necesidades de capacitación profesional sobre la perspectiva de organizaciones de reclutamiento de personal. Para las empresas especializadas en selección de personal, las organizaciones que más demandan sus servicios se agrupan en los sectores de Alimentos frescos y procesados; Construcción; Tecnología en hardware y software; y Productos farmacéuticos y químicos.

En segundo lugar, los sectores productivos con alta perspectiva de empleabilidad son los siguientes: Tecnología en hardware y software; Vehículos, automotores, carrocerías y partes; Turismo; Construcción; y Energías renovables, mismas que están acorde al objetivo de la matriz productiva del Ecuador y que tienen su importancia si se quiere pasar a ser una nación industrializada y productiva.

Igualmente, las nuevas ocupaciones y las que tienen dificultades de encontrar trabajadores competentes son: Profesionales científicos e intelectuales, quienes se encuentran con mayor frecuencia en el mundo académico; Trabajadores de los servicios y vendedores de comercios y mercados, cuya dificultad se debe primordialmente a la alta rotación que existe en este tipo de cargo, pues al hallarse un buen perfil suele ser fácilmente captado por la competencia, dejando vacantes en forma continua; y profesionales de nivel medio. Además es importante observar que en los centros de educación superior no existen programas académicos ligados específicamente a desarrollar este tipo de perfil, es por esta razón la importancia de la educación informal que prepara los profesionales en un área específica como las ventas. En muchos casos los profesionales de nivel medio tienen trayectorias de carrera no lineales, ya que intentan escoger cargos afines a sus estudios, pero en caso de no encontrar con facilidad utilizan caminos laberínticos que le permitan principalmente obtener recursos económicos aunque no sea su profesión. Este comportamiento no permite un proceso de especialización en la rama, ni tampoco una planificación laboral, que tiende a ser imprevista y azarosa.

Finalmente, las áreas académicas de mayor demanda o que se requiere de personal fueron identificadas como: Administración y legislación; Educación y Capacitación y Tecnologías de la información y comunicación. En estos ejes de conocimiento existen empresas específicas que preparan al profesional mediante cursos especializados o certificaciones, en cualquier caso es esencial que se tenga comunicación entre la empresa privada, pública u otros organismos con los centros de formación/educativos, sean estos formales e informales, para disminuir las brechas entre la educación y el perfil requerido por el mercado. 


\section{REFERÊNCIAS}

ADRIÁN, I.; PAULA, N. Guía metodológica para el estudio de las necesidades de formación e información de los usuarios o lectoresCiencias de la Información. [s.l: s.n.].

BARROS, R. M. da S. Diagnóstico de necessidades de formação. (Mestrado em Psicologia Social e das Organizações) - Instituo Universitario de Lisboa, 2011.

BOMFIN, D. Pedagogia no treinamento: correntes pedagógicas no ambiente de aprendizagem nas organizações. Rio de Janeiro: Qualitymark Editora Ltda, 2004.

BOOG, G. G. Manual de Treinamento e Desenvolvimentoo. São Paulo: Makron Books, 1994.

CRUZ, J. Formação Profissional em Portugal. Lisboa: Sílabo, 1997.

DA SILVA, M. F.; DA CONCEIÇÃO, F. A.; LEITE, M. M. J. Educação continuada: um levantamento de necessidades da equipe de enfermagem. Mundo da saúde, v. 32, n. 1, p. 47-55, 2008.

DALMAU, M. et al. Programa de educação profissional a distância ou presencial? A difícil escolha sobre qual o meio mais indicado para as empresas. Anais... ANAIS ENGEPI, 2001.

GIL, A. C. Administração de recursos humanos: um enfoque profissional. 3. ed. São Paulo: Atlas, 2001. HARRIS, B. A strategy for identifying the professional development needs of teachers: A report from New South Wales. Journal of In-Service Education, v. 26, n. 1, p. 25-47, 2000.

HARRIS, B. A strategy for identifying the professional development needs of teachers : a report from New South Wales Professional Development Needs of Teachers : a report from. Journal of In-Service Education, v. 26, jun. 2012, p. 37-41, 2006.

INA. Estudio de determinación de necesidades de capacitación y formación profesional para técnicos en mecatrónica. Costa Rica: [s.n.].

JACINTO, C. Nuevas lógicas en la formación profesional en Argentina Redefiniendo lo educativo, lo laboral y lo social. Perfiles Educativos, v. 37, n. 148, p. 120-137, 2015.

LACAVEX, M. A. Capacitación y adiestramiento para el trabajo en el Derecho Mexicano. Revista de Derecho (Valparaiso), n. 41, p. 85-105, 2013.

LEFCOVICH, M. La capacitación en la empresa Aspectos a tener en cuenta. 1. ed. Buenos Aires: El Cid Editor, 2009. 
MCGEHEE, W.; THAYER, P. W. Training in business and industry. New York: Wiley, 1961.

MEJÍA, Á.; MIRANDA, V.; QUIÑONES, E. Necesidades de Capacitación: Diagnóstico y Programación de Actividades. Colombia: [s.n.]. Disponível em: <http://mecicalidad.dafp. gov.co/documentacion/Componente Ambiente de Control/DiagnosticoNecesidadesCapacitacion.pdf>. Acesso em: 16 jul. 2012.

MENDOZA, A. Manual para determinar necesidades de capacitación. [s.l: s.n.].

MENESES, P. P. M.; ZERBINI, T. Levantamento de necessidades de treinamento: reflexões atuais. Análise-Revista de Administração da PUCRS, v. 20, n. 2, 2010.

MORAES, G. Desenvolvimento de um modelo para o levantamento de necessidades de treinamento e desenvolvimento de recursos humanos. JOUR-[s.I.] Florianópolis, SC, 2002.

ORTEGA, M. D. Capacitación les asegura el empleo. El Norte, p. 3, 5 jun. 2006.

PIECK GOCHICOA, E. Sentidos e incidencia de la capacitación técnica: visión desde los/as estudiantes. Revista mexicana de investigación educativa, v. 16, n. 48, p. 159-194, 2011.

POR, J.; AGUILAR, E. El diagnóstico de necesidades de capacitación. México: [s.n.].

RAMOS, J. M. G. La fórmación integral: objetivo de la Universidad (Algunas reflexiones sobre la educación en la Universidad). Revista Complutense de Educación, v. 2, n. 2, p. 323-335, 1991.

RODRÍGUEZ, F. A. Fectos del programa mexicano de capacitación en la duración del desempleo de sus participantes. El Trimestre Económico, a. 70, v. 278, n. 2, p. 275-314, 2003.

SERPE. Metodología para detectar necesidades de capacitación y determinar prioridades. Panamá: [s.n.]. Disponível em: <http://ceccsica.org/programas-accion/laboral/contenido_paises/Panama/Productos/Observatorio/Panama_Metodologia_Necesidades_Capacitacion.pdf>. Acesso em: 16 jul. 2012.

VERDIER, E. La educación y la formación a lo largo de la vida: una orientación Europea. Sociologie et Societes, v. 40, n. 1, p. 195-225, 2008.

VICEPRESIDENCIA DE LA REPÚBLICA DEL ECUADOR. Estrategia Nacional para el cambio de la Matriz Productiva. Quito: [s.n.]. Disponível em: <http://www.vicepresidencia.gob.ec/wp-content/uploads/2013/10/ENCMPweb.pdf>. Acesso em: 01 set. 2017. 